(C) 2006 The Japan Society of Applied Physics

\title{
Study of InGaN Multiple Quantum Dots by Metal Organic Chemical Vapor Deposition
}

\author{
Te-Chung WANG ${ }^{1,2 *}$, Hao-Chung KuO ${ }^{1}$, Tien-Chang $\mathrm{LU}^{1}$, Ching-En TsAI ${ }^{2}$, Min-Ying TsAI ${ }^{1}$, \\ Jung-Tsung $\mathrm{HSU}^{2}$ and Jer-Ren $\mathrm{YANG}^{3}$ \\ ${ }^{1}$ Institute of Electro-Optical Engineering, National Chiao Tung University, Hsinchu, Taiwan, Republic of China \\ ${ }^{2}$ Opto-Electronics and System Laboratories, Industrial Technology Research Institute, Hsinchu, Taiwan, Republic of China \\ ${ }^{3}$ Institute of Materials Science and Engineering, National Taiwan University, Taipei, Taiwan, Republic of China
}

(Received September 16, 2005; accepted December 22, 2005; published online April 25, 2006)

We reports a study of InGaN multiple quantum dot layers. Using the in-situ $\mathrm{SiN}_{x}$ treatment process, InGaN multiple quantum dot layers were successfully developed. The InGaN multiple quantum dot layers were constructed with $\mathrm{SiN}_{x}$ dot mask layers, InGaN dot layers, and $\mathrm{GaN}$ cap layers on a $2-\mu \mathrm{m}$-thick $\mathrm{GaN}$ underlying layer on a sapphire substrate. Optical properties including room temperature photoluminescence (PL), temperature dependent PL, and low power power-dependent PL were examined and discussed. The structure was also analyzed by transmission electron microscopy (TEM) and energy dispersive X-ray (EDX) line scan. [DOI: 10.1143/JJAP.45.3560]

KEYWORDS: InGaN QDs, MOCVD

\section{Introduction}

$\mathrm{GaN}$ and related materials are currently the subject of intense research due to their applications in laser diodes (LDs) and light-emitting diodes (LEDs) that operate between the ultraviolet and the visible regions. ${ }^{1)}$ InGaN/GaN quantum well (QW) structures have successfully been used as active layers in LEDs and LDs. ${ }^{1)}$ However, the threshold current density is high for LDs with InGaN QW structure. Having quantum dots (QDs) instead of QWs as the active layer is expected to improve the performance of LDs. LDs with QD structures in the active layer have been theoretically predicted to have superior characteristics, including lower threshold currents and narrow spectra. ${ }^{2}$ Moreover, because of the localization of carriers trapped at dislocations, QD structures have been expected to increase the efficiency of the luminescence of LDs and LEDs. ${ }^{3)}$ To ensure suitability for QD applications, QD layers with high spatial density and of uniform size must be grown. ${ }^{4)}$ Several approaches have been investigated for fabricating InGaN QDs, including the Stranski-Krastanow growth mode ${ }^{5,6)}$ and growth using an anti-surfactant. ${ }^{7,8)}$ The deposition of a silicon anti-surfactant or a $\operatorname{SiN}_{x}$ nano-mask alters the morphology of the AlGaN films from that of step flow to that of a three dimensional island, facilitating the formation of $\mathrm{GaN}^{7)}$ QDs and $\mathrm{InGaN}_{\mathrm{QDs}}{ }^{8)}$ on the AlGaN.

On the other hand, the properties of the InGaN-based red emission device are dominated by both the band-filling effect and the screening effect of the quantum confined stark effect (QCSE) due to the high indium composition and the large thickness of the InGaN well layer. Therefore, InGaN multiple quantum well light emitting devices are very difficult to achieve. However, the InGaN quantum dot devices possess the potential to overcome limitations to make the efficiency higher and the wavelength longer. Using the in-situ $\mathrm{SiN}_{x}$ treatment process that we have reported, ${ }^{9}$ red emission InGaN multiple quantum dots with ultra-high density have been successfully developed. In this report, we discuss the optical characteristics of InGaN red emission multiple quantum dots and their structure determined by TEM and EDX line scan.

*E-mail address: WangWang@itri.org.tw

\section{Experimental}

Despite many phenomenon of $\mathrm{InGaN}$ localization and segregation that have been reported, ${ }^{10,11)}$ the efficiency and intensity of high indium-content devices at room temperature are still quite low. It is very difficult to make a high efficiency red emission device. According to previous results, ${ }^{9}$ InGaN QD density of about $2 \times 10^{11} \mathrm{~cm}^{-2}$ has been achieved. We attempted to grow a five period, multistack structure to enhance the intensity of PL and decrease the deposition temperature of InGaN QDs to make the wavelength longer. The InGaN multiple quantum dot layers were constructed with $\mathrm{SiN}_{x}$ dot mask layers, InGaN dot layers, and $\mathrm{GaN}$ cap layers as shown in Fig. 1. At first, a 30nm-thick low-temperature GaN nucleation layer was grown on the $c$-plane of a sapphire substrate at $550{ }^{\circ} \mathrm{C}$ by metal organic chemical vapor deposition (MOCVD). Then, the reactor temperature was increased to $1000{ }^{\circ} \mathrm{C}$ to grow a 2$\mu \mathrm{m}$-thick $\mathrm{Si}$-doped $\mathrm{GaN}$ underlying layer, providing a step flow grown surface. An island-like random-formation $\mathrm{SiN}_{x}$ in-situ nano-mask was deposited on the n-type GaN underlying layer. During the $420 \mathrm{~s}$ treatment of the $\mathrm{SiN}_{x}$ nanomask, the flow rates of $\mathrm{NH}_{3}$ and diluted $\mathrm{Si}_{2} \mathrm{H}_{6}$ were $5 \mathrm{slm}$ and $50 \mathrm{sccm}$, respectively. The temperature was then ramped down to $750{ }^{\circ} \mathrm{C}$ to grow $2 \mathrm{~nm} \operatorname{In}_{x} \mathrm{Ga}_{1-x} \mathrm{~N}$ layers, and finally 3-nm-thick GaN was deposited as the cap layer. The temperature was increased $1000{ }^{\circ} \mathrm{C}$ to repeat the process

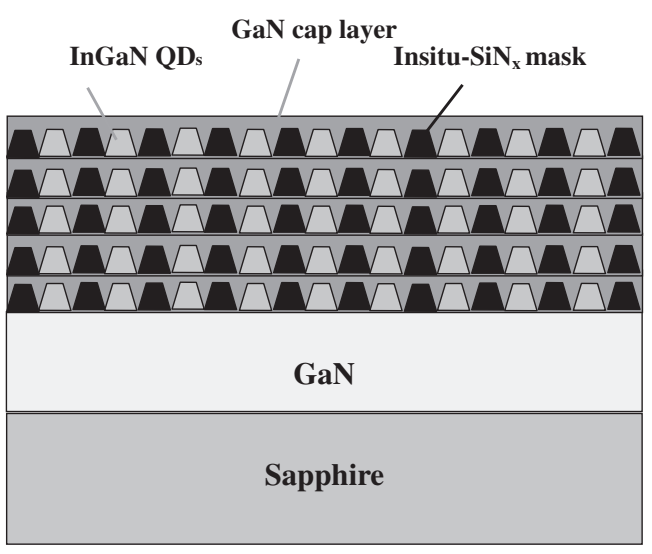

Fig. 1. Scheme for InGaN multiple quantum dot layers. 


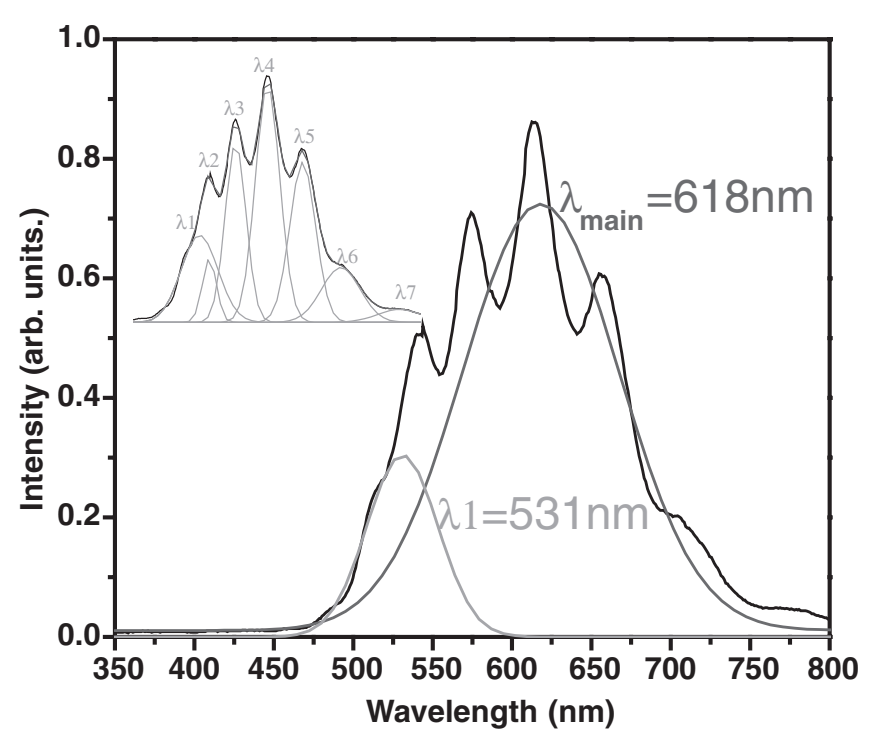

Fig. 2. Room temperature PL of InGaN MQDs.

and produce a five period multi-stack structure. During the growth of the InGaN layers, the vapor phase ratio TMIn/(TMIn + TEGa) was fixed higher than previous work at 0.40 . We investigated the optical properties of the InGaN multiple quantum dot layers by room-temperature PL using Accent rpm 2000, which contains a He-Cd $5 \mathrm{~mW}$ laser. Then we analyzed the InGaN MQD behavior by temperature-dependent PL and low-power power-dependent PL. Finally, we analyzed the structure by high resolution TEM and EDX line scan.

\section{Results and Discussion}

The room temperature PL of InGaN multiple quantum dot layers, shown in Fig. 2, was measured by Accent rpm 2000; the PL spectrum reveals a broadened emission from 480 to $800 \mathrm{~nm}$. The additional peaks between the blue emission and red emission are the results of Fabry-Perot interference fringes. By Gaussian peak fitting, the spectrum was divided into seven individual wavelengths, and the $\lambda_{2}$ to $\lambda_{7}$ indicated the same interference relationship. Consequently, the spectrum could be divided into two parts: emissions $\lambda_{1}$ at $531 \mathrm{~nm}$, and $\lambda_{\text {main }}$ at $618 \mathrm{~nm}$. The two emissions may be excited by different energy states. To further verify the origin of the emission, the bandgap of InGaN MQDs was calculated based on the equation provide by $\mathrm{Wu}$ et al.:12)

$$
\begin{aligned}
E_{\mathrm{g}}\left(\operatorname{In}_{x} \mathrm{Ga}_{1-x} \mathrm{~N}\right)= & E_{\mathrm{g}}(\mathrm{InN})_{x}+E_{\mathrm{g}}(\mathrm{GaN}) \\
& \times(1-x)-1.42 x(1-x)
\end{aligned}
$$

where $x$ is the indium concentration, and $E_{\mathrm{g}}(\operatorname{InN})$ and $E_{\mathrm{g}}(\mathrm{GaN})$ represent the bandgap energies of $\mathrm{InN}(0.77 \mathrm{eV})$ and $\mathrm{GaN}(3.42 \mathrm{eV})$, respectively. Following the calculation, the indium compositions of the two energy states are 0.30 and 0.40 . However, the high resolution X-ray diffraction measurement of the MQD structure is too broad to analyze, and this yields the same result as reported, ${ }^{11)}$ for phase separation in InGaN/GaN multiple quantum wells. The high temperature of the $\mathrm{SiN}_{x}$ treatment step may lead the formation of different InGaN phases and the lower temperature in-situ $\mathrm{SiN}_{x}$ nano-mask technique should be studied further in the future. The red emission at room temperature

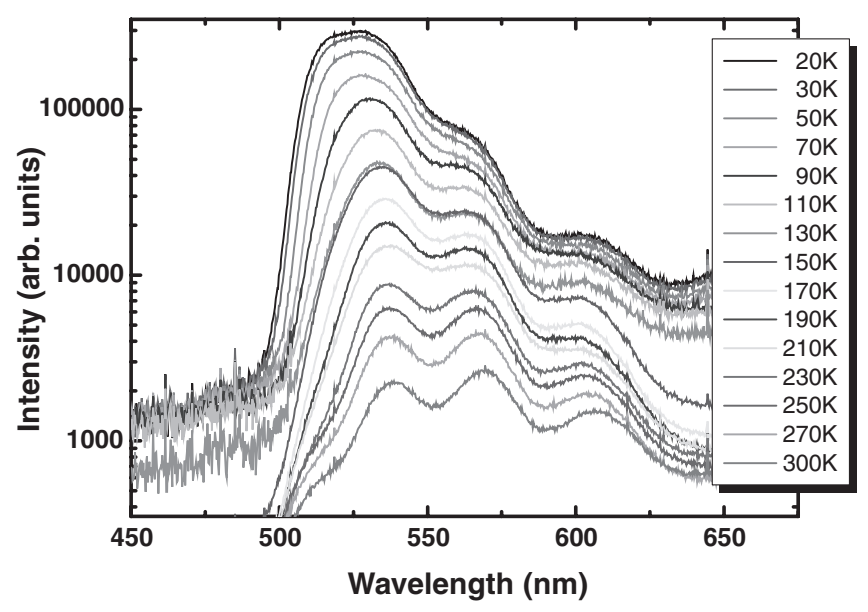

Fig. 3. Temperature-dependent PL of InGaN MQDs.

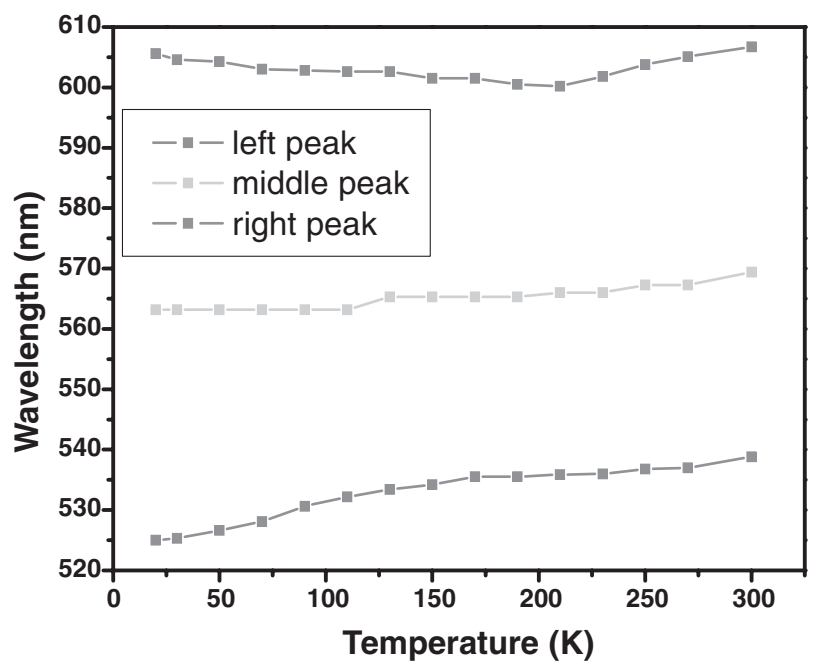

Fig. 4. Trends of main peak shifts in the temperature-dependent PL of InGaN MQDs.

is quite strong; it is evidence that ultra-high density InGaN multiple quantum dots have the potential to be used in red emitting devices.

Figure 3 shows the temperature-dependent PL of InGaN MQDs. Three different PL peaks are present, and all intensities increase as temperature decreases. By connecting the main peak positions, the trends of the three peaks shift with the temperature as shown in Fig. 4. The left peak is obviously blue shifted from 540 to $525 \mathrm{~nm}$ as the temperature decreases like a general uniform quantum well. In comparison with the structure, this could be the wetting layer of quantum dots generated by InGaN phase separation. The middle peak is stable around $567 \mathrm{~nm}$; this may come from the nature of defects in $\mathrm{GaN}$ referred to as yellow luminescence. Because the power density of the exciting laser is higher than rpm 2000, deeper natural defects were excited. The right peak above $600 \mathrm{~nm}$ is blue shifted first and red shifted as the temperature decrease. This luminescence exhibits an "S-shaped" emission shift in position with temperature. The redshift-blueshift phenomenon was first explained by $\mathrm{Cho}^{13)}$ in terms of inhomogeneity and carrier localization in InGaN. Consequently, we may conclude the in-situ $\mathrm{SiN}_{x}$ treatment of InGaN multiple quantum dot layers 


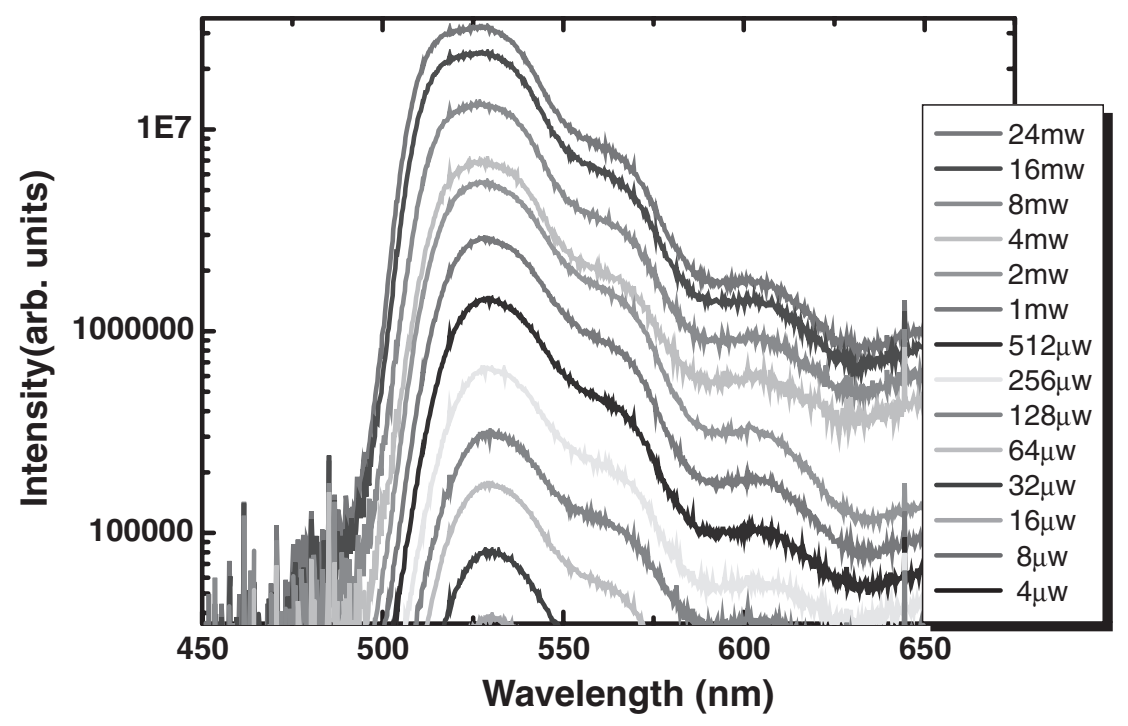

Fig. 5. Low-power power-dependent PL of InGaN QDs at $20 \mathrm{~K}$.

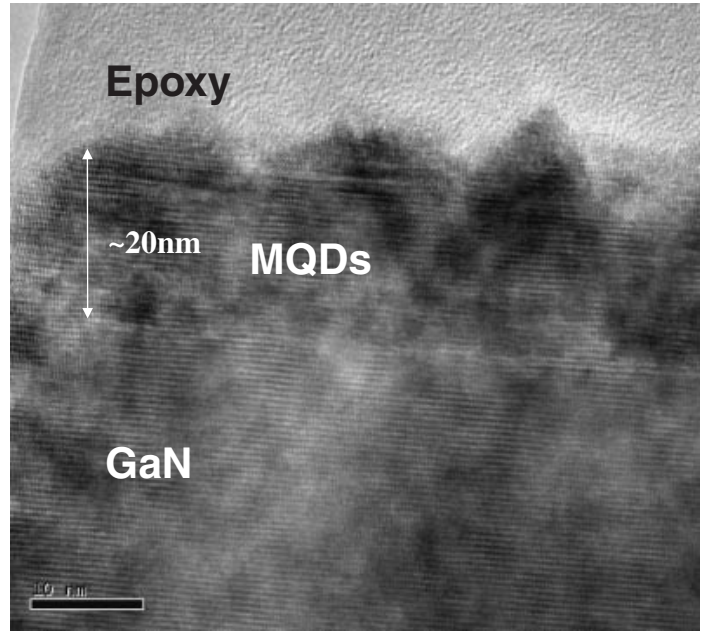

Fig. 6. High resolution TEM image of InGaN MQDs.

causes the same behavior of carrier localization, and the wavelength could be pushed toward a red emission. At the same time, ultra-high density of InGaN QDs of approximately $2 \times 10^{11} \mathrm{~cm}^{-2}$ also could be achieved by this procedure. As Fig. 5 shows, low-power power-dependent PL spectra of InGaN MQDs at $20 \mathrm{~K}$ have three emission peaks as well, and all intensities increase as power increases. The left peak is blue shifted from 530 to $525 \mathrm{~nm}$ as power increases, and the behavior is still like that of a wetting layer of quantum dot; the middle peak demonstrates yellow luminescence. The position of the right peak is almost the same as the rise in exciting power. The phenomenon shows the emission generated by InGaN MQDs was subjected to a very low piezoelectric field, and it may be possible to make a red emitting device by this method. Figure 6 shows the high resolution TEM image of InGaN MQDs. An obvious interface exists between the high temperature GaN underlying layer and the five pairs of MQDs, which consist of 2nm-thick InGaN layers and 3-nm-thick cap layers. Unfortunately, the boundary between $\mathrm{InGaN}_{\text {and }} \mathrm{SiN}_{x}$ can not be identified in this image. Because of randomness in size and in position, isolated InGaN QDs and $\mathrm{SiN}_{x}$ domains were not

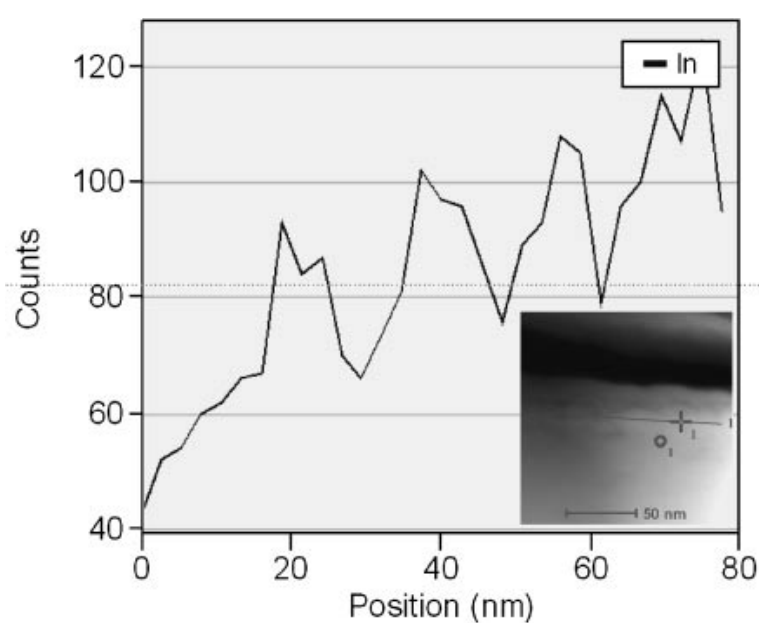

Fig. 7. EDX line scan in the horizontal direction of InGaN MQDs.

easily found in the cross-sectional view. As the EDX line scan in the horizontal direction of MQDs shows in Fig. 7, the indium composition fluctuates in regular size about $20 \mathrm{~nm}$, and the size is almost the same as indicated by the AFM data in previous work. ${ }^{9)}$ It is strong evidence toward that establishing $\mathrm{SiN}_{x}$ is suitable to use as a nano-mask to control the InGaN QD size. On the other hand, as the EDX line scan in the vertical direction of the MQDs shows in Fig. 8, the indium composition does not obviously fluctuate. This may result from indium diffusing into the GaN barrier layer during high temperature $\operatorname{SiN}_{x}$ treatment. The low temperature in-situ $\mathrm{SiN}_{x}$ nano-mask technique should be studied further, and low the temperature $\mathrm{p}-\mathrm{GaN}$ contact layer should be made available for LED device structure or else the high temperature will damage the dot layers.

\section{Conclusions}

An in-situ $\mathrm{SiN}_{x}$ nano-mask technique has been successfully used for InGaN MQD growth by MOCVD. A red emission from InGaN MQDs was demonstrated at lower growth temperatures and higher indium/gallium ratios. InGaN MQDs based on a $\mathrm{SiN}_{x}$ in-situ nano-mask may be under the influence of a low piezoelectric field, and it may be 


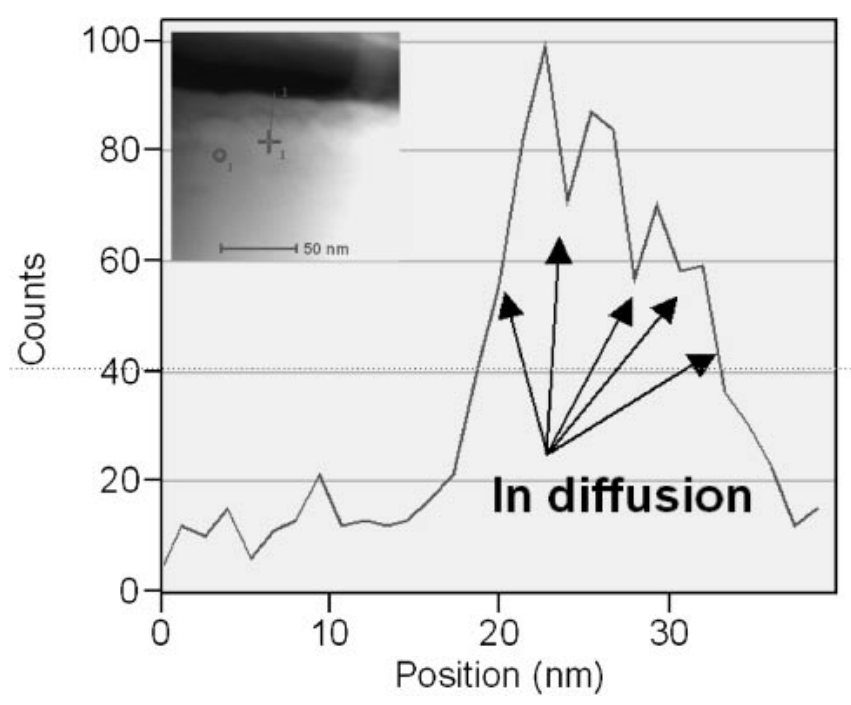

Fig. 8. EDX line scan in the vertical direction of InGaN MQDs.

possible to use this phenomenon to develop red LEDs. During MQD growth, $\mathrm{SiN}_{x}$ is stable enough to confine the InGaN QDs. However, the indium in InGaN QDs diffuses easily into the $\mathrm{GaN}$ barrier layer during the high temperature growth of $\mathrm{SiN}_{x}$. Further study of the low temperature in-situ $\mathrm{SiN}_{x}$ nano-mask technique should be carried out.

\section{Acknowledgement}

The authors thank the Ministry of Economic Affairs, Taiwan, Republic of China, for financial support.

1) S. Nakamura and G. Fasol: The Blue Laser Diode (Springer, Heidelberg, 1997).

2) C. Adelmann, J. Simon, G. Feuillet, N. T. Pelekanos and G. Fishman: Appl. Phys. Lett. 76 (2000) 1570.

3) B. Damilano, N. Grandjean, S. Dalmasso and J. Masies: Appl. Phys. Lett. 75 (1999) 3751.

4) D. Bimberg, M. Grundmann and N. N. Ledentsov: Quantum Dot Heterostructures (Wiley, Chichester, 1999).

5) K. Tachibana, T. Someya and Y. Arakawa: Appl. Phys. Lett. 74 (1999) 383.

6) B. Daudin, F. Widmann, G. Feuillet, Y. Samson, M. Arlery and J.-L. Rouvière: Phys. Rev. B 56 (1997) R7069.

7) S. Tanaka, S. Iwai and Y. Aoyagi: Appl. Phys. Lett. 69 (1996) 4096.

8) H. Hirayama, S. Tanaka, P. Ramvall and Y. Aoyagi: Appl. Phys. Lett. 72 (1998) 1736.

9) R. C. Tu, C. J. Tun, C. C. Chuo, B. C. Lee, C. E. Tsai, T. C. Wang, J. Chi, C. P. Lee and G. C. Chi: Jpn. J. Appl. Phys. 43 (2004) 264.

10) Fei Chen, A. N. Cartwright, C. Liu and I. M. Watson: Phys. Status Solidi C 2 (2005) 2787.

11) M. D. McCluskey, L. T. Romano, B. S. Krusor, D. P. Bour, N. M. Johnson and S. Brennan: Appl. Phys. Lett. 72 (1998) 1730.

12) J. Wu, W. Walukiewicz, K. Yu, J. Ager III, E. Haller, H. Lu and W. Schaff: Appl. Phys. Lett. 80 (2002) 4741.

13) Y. H. Cho, G. H. Gainer, A. J. Fischer, J. J. Song, S. Keller, U. K. Mishra and S. P. DenBaars: Appl. Phys. Lett. 73 (1998) 1370. 